\title{
ANÁLISIS MORFOMÉTRICO DE MICROCUENCAS AFECTADAS POR FLUJOS DE DETRITOS BAJO PRECIPITACIÓN INTENSA EN LA QUEBRADA DE CAMIÑA, NORTE GRANDE DE CHILE*
}

\author{
MORPHOMETRIC ANALYSIS OF MICRO WATERSHEDS AFFECTED FOR \\ DEBRIS FLOWS CAUSED BY HEAVY RAINS IN THE CAMIÑA VALLEY, \\ NORTHERN OF CHILE
}

\author{
Mónica Meza Aliaga**, Alan Rodríguez Valdivia ${ }^{* * *}$, Oscar Corvacho Ganahín ${ }^{* * *}$ \\ y Alejandro Tapia Tosetti**
}

\begin{abstract}
Se analizan los distintos parámetros morfométricos de las microcuencas Cuisama, Camiña (1 y 2), Chapiquilta (1, 2 y 3), Yala-Yala ( 1,2 y 3), Apamilca (1 y 2) y Altuza, previo a los flujos de detritos acontecidos el 13 de marzo de 2012 en la quebrada de Camiña localizada en el Norte de Chile. Se utilizaron métodos cuantitativos que permitieron obtener distintos parámetros morfométricos de las cuencas analizadas. Entre los resultados destacan los altos valores de las densidades de drenaje, tiempos de concentración muy reducidos, formas compactas de las cuencas y curvas hipsométricas que determinaron una tendencia a ciclos erosivos inestables. Todas ellas características favorables para el desarrollo de flujos de detritos y fenómenos aluvionales.
\end{abstract}

Palabras claves: Quebrada de Camiña, morfometría de cuencas hidrográficas, análisis hipsométrico.

Morphometric parameters of the various micro-basins Cuisama, Camiña (1 and 2), Chapiquilta (1, 2 and 3), Yala Yala (1, 2 and 3), Apamilca (1 and 2) and Altuza prior analyzes to debris flows that occurred on March 13, 2012 in the gorge of Camiña located in northern Chile. Quantitative methods, we have obtained different watershed morphometric parameters analyzed were used. Highlights included the high values of drainage density, very reduced concentration, compact forms of watersheds and hypsometric curves determined a tendency to unstable erosion cycles. All favorable for the development of debris flows and alluvial phenomena characteristics.

Key words: Camiña ravine, watershed morphometry, hypsometric analysis.

\section{Introducción}

Diversos autores coinciden en que las precipitaciones y sus características serían uno de los principales detonantes de los movimientos en ladera (Almorox; Llano, 1975; Walker y Shiels, 2012). Las lluvias constituyen el más agresivo y principal detonante de procesos de remoción en masa en el planeta y debido a las consecuencias del cambio climático y calentamiento global se vaticina un alteración de las condiciones normales del clima; lo que significa que procesos como el ciclo hidrológico y eventos meteorológicos, como la precipitación, experimentarán una distorsión en su comportamiento habitual (Lacasse y Nadim, 2008), extremando fenómenos asociados como sequías, inundaciones y fenómenos de remoción en masa.

La incidencia de fenómenos hidrometeoros como la lluvia en la provocación de deslizamiento alrededor del mundo cobra tal importancia en algunas regiones del planeta, que incluso en zonas con mayor aridez se convierte en el principal detonante de este tipo de procesos. Al respecto, Llano (1975) considera relevante las fluctuaciones temporales que puede presentar la precipitación en la generación de eventos de deslizamiento, indicando que la alternancia entre lluvias violentas y sequedad

\footnotetext{
* Investigación desarrollada en el marco del proyecto FONDECYT No 1120204 y del Centro de Investigaciones y Vulnerabilidades Socionaturales -CIVDES-. Agradecimientos Convenio de Desempeño UTA-MINEDUC.

** Universidad de Tarapacá, Departamento de Ciencias Históricas y Geográficas, Arica, Chile. Correos electrónicos: msmezaa@uta.cl; atapia@uta.cl

*** Investigador independiente. Correos electrónicos: alanrodriguezvaldivia@gmail.com; geoscar.uta@gmail.com
} 
prolongada puede ser letal, considerando que una lluvia de mediana intensidad podría detonar desastres de grandes proporciones en función de las condiciones físicas de las cuencas receptoras.

El 13 de marzo del 2012 la quebrada de Camiña fue afectada por lluvias intensas que produjeron movimientos en masa. Esto fue causado por lluvias de carácter convectivo, acompañadas de nubes con marcado desarrollo vertical correspondientes al evento climático denominado Invierno Altiplánico o Lluvias Estivales (Aceituno 1993). La corta duración de estas precipitaciones en aproximadamente 2 horas fue suficiente para que se activaran las microcuencas que componen la parte alta de la quebrada, lo que propició que se desarrollaran movimientos en masa, principalmente de tipo flujo de detritos (Méndez et al. 2013). Los flujos fueron capaces de desplazar una gran cantidad de material detrítico desde las partes altas de las vertientes norte y sur de la quebrada, afectando a aquellas localidades ubicadas en antiguas unidades de acumulación.

El reporte del 22 de marzo de 2012 emitido por ONEMI (2012b) deja de manifiesto que el martes 12 de marzo de 2012 se declara alerta roja para la provincia del Tamarugal de la Región de Tarapacá por lluvias estivales. El mismo reporte señala que el 14 de marzo de 2012 se declara como afectadas por la catástrofe a las comunas de Camiña y Huara, y el mismo día se define situación de emergencia agrícola.

De acuerdo con los reportes emitidos por ONEMI (2012: a, b y c), las localidades más afectadas por el evento climático fueron Camiña, Chapiquilta y Yala-Yala. Del total de viviendas afectadas destacan las localidades de Camiña (94),

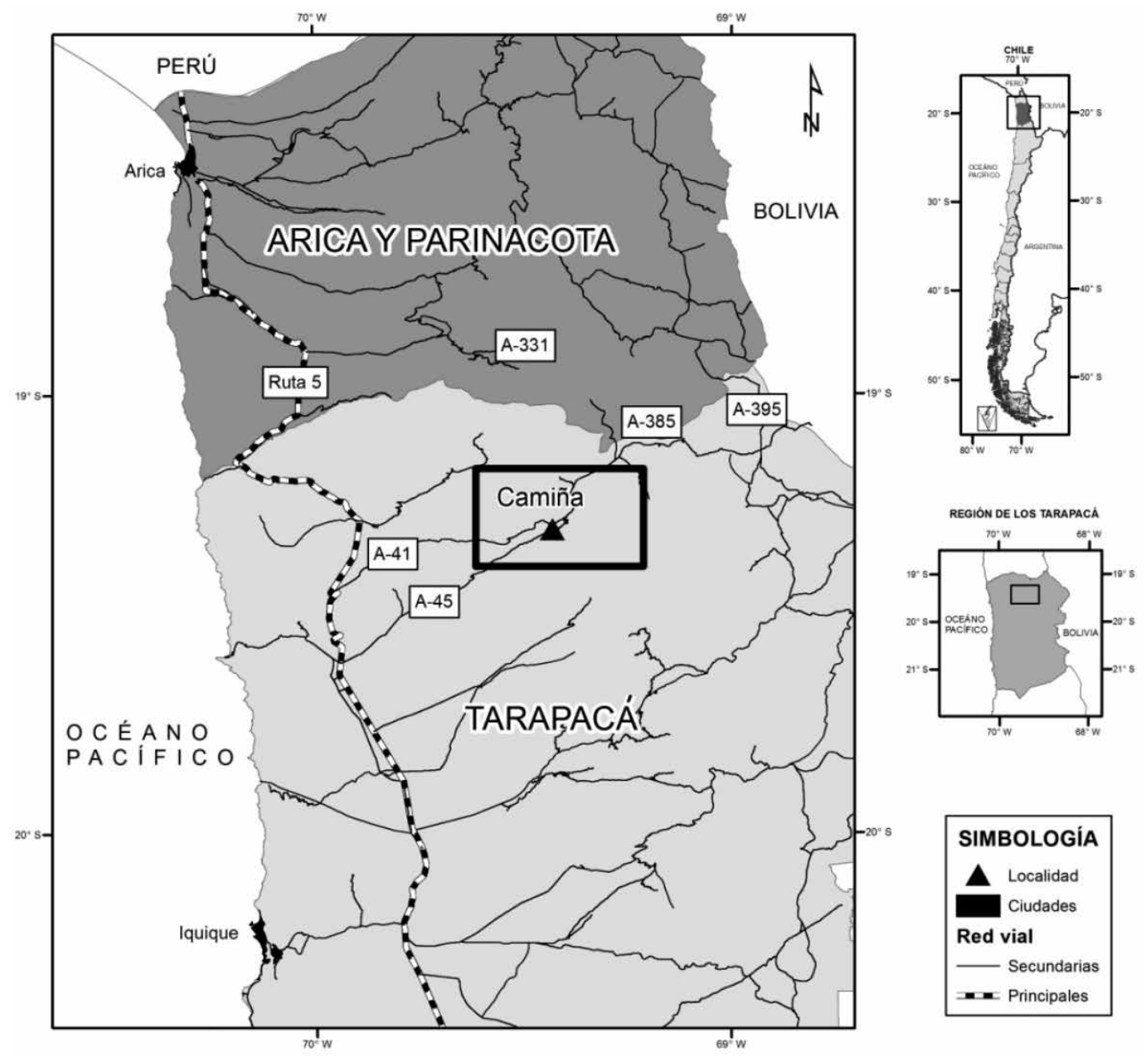

Figura 1. Área de estudio. Fuente: Elaboración propia. 
Chapiquilta (84), Yala-Yala (29) y Apamilca (2); mientras el total de personas albergadas arrojó 180 personas.

El mayor impacto en las localidades afectadas lo sufrió la infraestructura urbana, las áreas de cultivo y el sistema de riego, no habiendo pérdida de vidas humanas; sin embargo y considerando la recurrencia de estos eventos en el actual escenario de cambio climático es preciso un conocimiento acabado sobre la dinámica geomorfológica y morfométrica de las microcuencas que componen la quebrada. Este conocimiento, como proponen Gaillard y Mercer, 2013 y Gaillard et al., 2013, es parte de una ruta que integra conocimiento, acción y toma de decisiones para la reducción de desastres.

En consecuencia, el trabajo pretende determinar las condiciones morfométricas antes de los eventos naturales, de las microcuencas en que se produjeron los flujos de detritos (Figura 2) con el propósito de determinar si dichas condiciones favorecieron la ocurrencia de fenómenos de deslizamiento en la parte alta de la quebrada de Camiña desde Cuisama hasta Altuza.

\section{Metodología}

Para el análisis se seleccionaron microcuencas bajo los siguientes dos criterios:

1. Manifestación de flujos de detritos

2. Infraestructura aldeana afectada

Ambas características fueron reconocidas tanto en campo como por técnicas de fotointerpretación utilizando fotografías aéreas escala 1:50.000 del vuelo GEOTEC 1997 del Servicio Aerofotogramétrico -SAF-.

Se seleccionaron 12 microcuencas: una en Cuisama, dos en Camiña, tres en Chapiquilta, tres en Yala-Yala, dos en Apamilca y una en Altuza (Figura 2); todas con graves daños a la infraestructura pública y privada de las localidades asentadas en la parte alta de la quebrada localizadas sobre la Flexura Moquella.

\section{Parámetros morfométricos}

Los parámetros morfométricos fueron obtenidos a partir de un trabajo conjunto entre ecuaciones

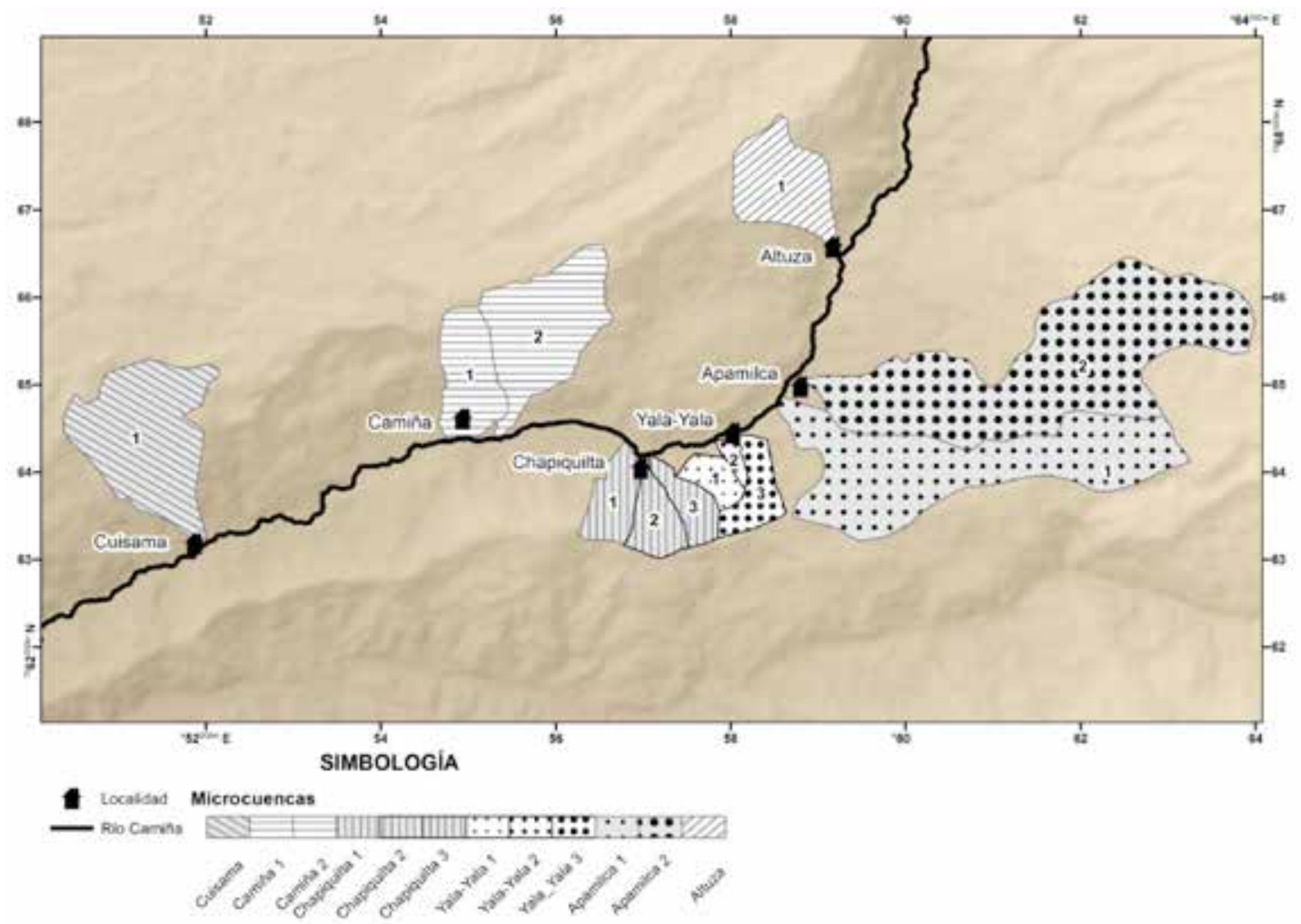

Figura 2. Microcuencas analizadas. Fuente: elaboración propia. 
hidrológicas y Sistemas de Información Geográfica -SIG-mediante el software ArcMap 10.1 (Figura 3). El SIG posibilitó el análisis espacial de los datos y la delimitación de las microcuencas estudiadas. Las ecuaciones hidrológicas por su parte permitieron determinar los parámetros morfométricos del estudio, de estos se consideraron: área, perímetro, desnivel, pendiente promedio de cada cuenca, longitud del cauce principal, longitud de la red hídrica, coeficiente de compacidad, densidad de drenaje y tiempo de concentración de las cuencas.

Los parámetros morfométricos fueron levantados a partir de un Modelo Digital de Terreno (MDT) ASTER GDEM correspondiente a octubre del 2011, cuyo pixel cubre una superficie de $900 \mathrm{~m}^{2}$. A partir de dicho modelo se crearon curvas de nivel con una equidistancia de $50 \mathrm{~m}$ y un modelo de elevación en tres dimensiones. En ArcMap 10.1 se utilizaron principalmente herramientas de análisis hidrológico, de la extensión Herramientas de Análisis Espacial, como flujo acumulado, dirección de flujo y orden de corrientes. Con esta herramienta se obtuvieron los parámetros de área, perímetro, desnivel, pendiente promedio de cada cuenca, longitud del cauce principal y longitud de la red hídrica.

Para obtención de los últimos tres parámetros se utilizaron las siguientes ecuaciones:

Coeficiente de Compacidad de Gravelius (1.1): Es la razón entre el perímetro de la cuenca y el área de la cuenca.

$$
K_{c}=0.28 \frac{P}{\sqrt{A}}
$$

Donde:

$\mathrm{K}_{\mathrm{c}}=$ Coeficiente de Compacidad

$\mathrm{P}=$ Perímetro de la cuenca $(\mathrm{km})$

$\mathrm{A}=$ Área de la cuenca $\left(\mathrm{km}^{2}\right)$

Los valores obtenidos de este índice nunca serán inferiores a 1, y mientras su grado de aproximación

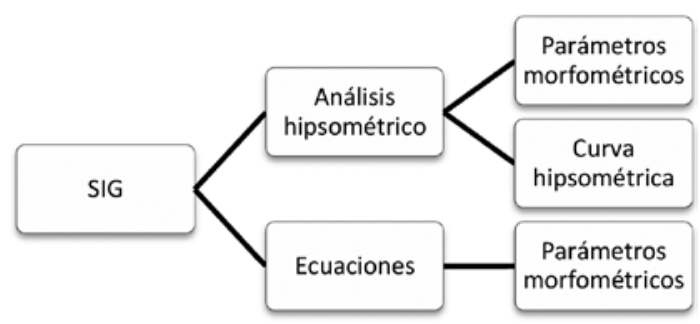

Figura 3. Planteamiento metodológico de la investigación. Fuente: elaboración propia. sea más cercano a 1 indicará una tendencia a concentrar grandes volúmenes de agua de escurrimientos (Instituto Nacional de Ecología -INE-, 2004).

Tabla 1. Clases de valores de compacidad (INE,2004).

\begin{tabular}{cl}
\hline Rango de Kc & \multicolumn{1}{c}{ Clases de compacidad } \\
\hline $1-1.25$ & Redonda a oval redonda \\
$1.25-1.50$ & De oval redonda a oval oblonga \\
$1.50-1.75$ & De oval oblonga a rectangular oblonga \\
\hline
\end{tabular}

Densidad de drenaje (1.2): Corresponde a la longitud total de los cauces dentro de una cuenca, divida por el área total de la cuenca (Horton 1945; Campos 1992).

$$
D_{d}=\frac{\Sigma L}{A}
$$

Donde:

$D_{d}=$ Densidad de drenaje $\left(\mathrm{km} / \mathrm{km}^{2}\right)$

$\mathrm{L}=$ Longitud total de los cauces $(\mathrm{km})$

$\mathrm{A}=$ Área total de la cuenca $\left(\mathrm{km}^{2}\right)$

Según el INE, 2004, valores próximos o superiores a $0,5 \mathrm{~km} / \mathrm{km}^{2}$ indican la eficiencia de la red de drenaje.

Tiempo de Concentración (1.3): Se entiende como el tiempo que demoran todos los puntos de una cuenca en aportar agua de escorrentía, de forma simultánea, al punto de salida de la cuenca (Ibáñez et al.: 3; Sharifi y Hosseini, 2011). Para obtener este parámetro se utilizó la ecuación California Culvert Practice (1955), obtenida de Li y Chibber (2008):

$$
T_{c}=60\left(11.9 \frac{L^{3}}{H}\right)^{0.385}
$$

Donde:

$\mathrm{T}_{\mathrm{c}}=$ Tiempo de concentración en minutos

$\mathrm{L}=$ Longitud del curso de agua principal (millas)

$\mathrm{H}=$ Desnivel de la cuenca (pies)

\section{Análisis hipsométrico}

El análisis hipsométrico es el estudio de la distribución del área de superficie del terreno, o la sección transversal del área, de una masa de tierra respecto de la elevación (Strahler, 1952). También se puede entender como la representación gráfica de las elevaciones del terreno en función de las superficies 
correspondientes (Campos, 1992). El gráfico obtenido representa en el eje $X$ las superficies relativas y en el eje $\mathrm{Y}$ las alturas relativas (Figura 4). El área relativa es la fracción del área (a) sobre esa cota (h) respecto del área total (A); mientras que la altura relativa es la correspondiente a la cota $(\mathrm{h})$, dividida por la altura de la cuenca (H) (Quezada et al., 2010).

La figura 4.b corresponde a la representación gráfica de los datos obtenidos, donde aparecen tres curvas que significan el ciclo de erosión de la cuenca. La curva 1 corresponde a una etapa de desequilibrio erosivo y representa una cuenca geológicamente joven. La curva 2 corresponde a una etapa de equilibrio erosivo y representa una cuenca geológicamente madura. La curva 3 representa una cuenca erosionada y en estado de senectud (Campos, 1992).

\section{Resultados}

\section{Análisis morfométrico}

El 58,3\% de las microcuencas presentan un tamaño muy reducido (no superiores a $1 \mathrm{~km}^{2}$ ), entre las que se encuentran las cuencas de Chapiquilta (1, 2 y 3$)$, Yala-Yala (1, 2 y 3 ) y Camiña $1 ;$ el $25 \%$ presentan un tamaño intermedio (entre 1 a $3 \mathrm{~km}^{2}$ ), agrupándose en esta las microcuencas de Cuisama, Altuza y Camiña 2; el 16,67\% restante corresponden a las de mayor superficie (entre 3 y $5 \mathrm{~km}^{2}$ ), representadas por las microcuencas de Apamilca (1 y 2). En cuanto al perímetro, este aumenta en cada microcuenca a medida que aumenta la superficie de cada una de ellas, siendo el valor más bajo para Yala-Yala 2 y el mayor para Apamilca 2 (Tabla 2).

El desnivel obtenido determina importantes diferencias de altura en las microcuencas analizadas; es así como Cuisama, Camiña 2, Apamilca 1 y 2 y Altuza presentan desniveles sobre los $600 \mathrm{~m}$, y contrariamente las microcuencas de Yala-Yala son las que poseen los desniveles más bajos (300 m o menos).

En cuanto a la longitud de la red hídrica se ha determinado que el $66,67 \%$ de las microcuencas analizadas poseen una longitud inferior a los 10 $\mathrm{km}$, mientras que el resto presenta longitudes que varían entre los $16 \mathrm{~km}$ y hasta los $38 \mathrm{~km}$. De igual manera, los datos obtenidos de la longitud del cauce principal continúan reflejando diferencias importantes entre las microcuencas de mayor y menor superficie. Esto se puede ejemplificar por las significativas desigualdades entre las microcuencas de Yala-Yala 1 y 2 y Apamilca 1 y 2 , ya que estas últimas presentan las mayores longitudes de cauce principal del total analizado (superiores a $6 \mathrm{~km}$ ) y las primeras, como se aprecia en la Tabla 2, tienen longitudes muy reducidas (inferiores a $1 \mathrm{~km}$ ).

Las pendientes promedio de las microcuencas varían entre el $26 \%$ y $51 \%$, siendo los valores más altos los de Cuisama y Altuza. Importante es el promedio que obtuvo Camiña 1 y 2 , alcanzando una pendiente promedio del $42 \%$, lo que en principio explicaría la violencia de los flujos que además estarían asociados a los parámetros de superficie y red hídrica. Los casos de Chapiquilta y Yala-Yala son importantes debido a que las pendientes se encuentran en rangos de entre el $29 \%$ y $40 \%$, sin embargo se debe tener en cuenta la superficie de estas microcuencas, pues corresponden a las más pequeñas del total estudiado.

El índice de Gravelius permitió establecer que el $33,33 \%$ de las microcuencas analizadas poseen forma redonda a oval redonda, $50 \%$ forma oval redonda a oval oblonga y $16,67 \%$ forma oval
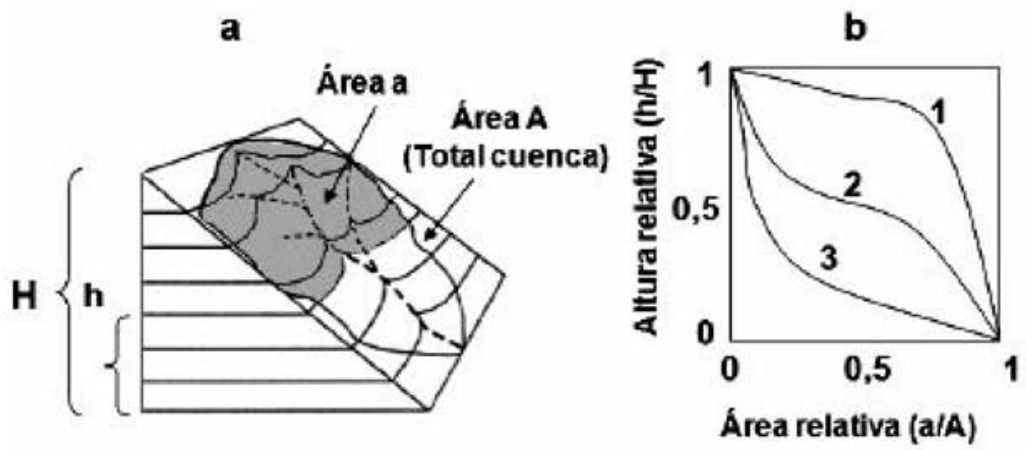

Figura 4. Esquema de datos para construir la curva hipsométrica. Fuente: modificado de Quezada, 2010. 
Tabla 2. Parámetros morfométricos de las microcuencas analizadas en la quebrada de Camiña. Fuente: elaboración propia.

\begin{tabular}{lccccccccc}
\hline Microcuenca & $\begin{array}{c}\text { Área } \\
(\mathrm{km})\end{array}$ & $\begin{array}{c}\text { Perímetro } \\
(\mathrm{km})\end{array}$ & $\begin{array}{c}\text { Desnivel } \\
(\mathrm{m})\end{array}$ & $\begin{array}{c}\text { Pendiente } \\
\text { promedio de la } \\
\text { cuenca }(\%)\end{array}$ & $\begin{array}{c}\text { Longitud } \\
\text { cauce } \\
\text { principal } \\
(\mathrm{km})\end{array}$ & $\begin{array}{c}\text { Longitud red } \\
\text { hídrica }(\mathrm{km})\end{array}$ & $\begin{array}{c}\text { Índice } \\
\text { Gravelius }\end{array}$ & $\begin{array}{c}\text { Densidad de } \\
\text { drenaje }\end{array}$ & $\begin{array}{c}\text { Tiempo de } \\
\text { concentración } \\
\text { (minutos) }\end{array}$ \\
\hline Cuisama & 2.18 & 6.96 & 711.17 & 50.65 & 3.08 & 22.16 & 1.30 & 10.16 & 16.60 \\
Camiña 1 & 0.88 & 4.02 & 648.00 & 42.04 & 2.04 & 6.49 & 1.20 & 7.34 & 10.74 \\
Camiña 2 & 1.74 & 6.39 & 735.00 & 42.45 & 3.20 & 16.32 & 1.30 & 9.36 & 17.19 \\
Chapiquilta 1 & 0.50 & 3.23 & 428.66 & 40.66 & 1.36 & 3.56 & 1.30 & 7.12 & 7.92 \\
Chapiquilta 2 & 0.45 & 3.00 & 351.45 & 36.19 & 1.21 & 3.23 & 1.20 & 7.18 & 7.40 \\
Chapiquilta 3 & 0.37 & 2.97 & 304.07 & 29.18 & 1.33 & 2.26 & 1.40 & 7.02 & 8.80 \\
Yala-Yala 1 & 0.27 & 2.21 & 198.57 & 32.46 & 0.89 & 1.71 & 1.20 & 6.34 & 6.44 \\
Yala-Yala 2 & 0.10 & 1.50 & 180.00 & 32.38 & 0.68 & 0.79 & 1.30 & 8.21 & 4.90 \\
Yala-Yala 3 & 0.47 & 3.57 & 300.00 & 31.37 & 1.68 & 3.69 & 1.50 & 7.87 & 11.47 \\
Apamilca 1 & 3.50 & 12.19 & 767.71 & 26.61 & 6.06 & 26.02 & 1.80 & 7.43 & 35.36 \\
Apamilca 2 & 4.98 & 13.70 & 860.00 & 33.23 & 6.26 & 38.03 & 1.70 & 7.64 & 35.09 \\
Altuza & 1.12 & 4.63 & 687.28 & 44.67 & 2.48 & 9.68 & 1.20 & 8.65 & 13.12 \\
\hline
\end{tabular}

oblonga a rectangular oblonga. En esta última destacan las microcuencas de Apamilca. La densidad de drenaje es para todas las microcuencas muy alta, por sobre los $6,34 \mathrm{~km} / \mathrm{km}^{2}$, siendo el límite los $10,16 \mathrm{~km} / \mathrm{km}^{2}$.

El tiempo de concentración (Tc) entregó resultados inquietantes, ya que los tiempos son muy reducidos para la mayoría de las microcuencas (Figura 5). Del total de microcuencas analizadas el $41,67 \%$ no superan los 10 minutos, entre las que se encuentran Chapiquilta 1, 2 y 3 y Yala-Yala 1 y 2. El 41,67\% pertenece a tiempos de entre 10 y 20 minutos, rango en donde se categorizan Cuisama, Camiña 1 y 2, Yala-Yala 3 y Altuza. Finalmente las microcuencas de Apamilca alcanzaron tiempos de concentración de 35 minutos.

El análisis hipsométrico entregó resultados interesantes respecto del ciclo erosivo y tipo de cuencas que se analizaron. Las Figuras 6 y 7 muestran una importante aglomeración de curvas hipsométricas en la etapa de desequilibrio y equilibrio, cuyos límites permiten interpretar que las microcuencas de Altuza y Yala-Yala 3 poseían una etapa erosiva muy próxima al desequilibrio, mientras que por el contrario, la curva hipsométrica de Camiña 1 es la más próxima al ciclo de cuenca erosionada. De la misma interpretación se obtiene que la gran mayoría de la microcuencas corresponden a cuencas

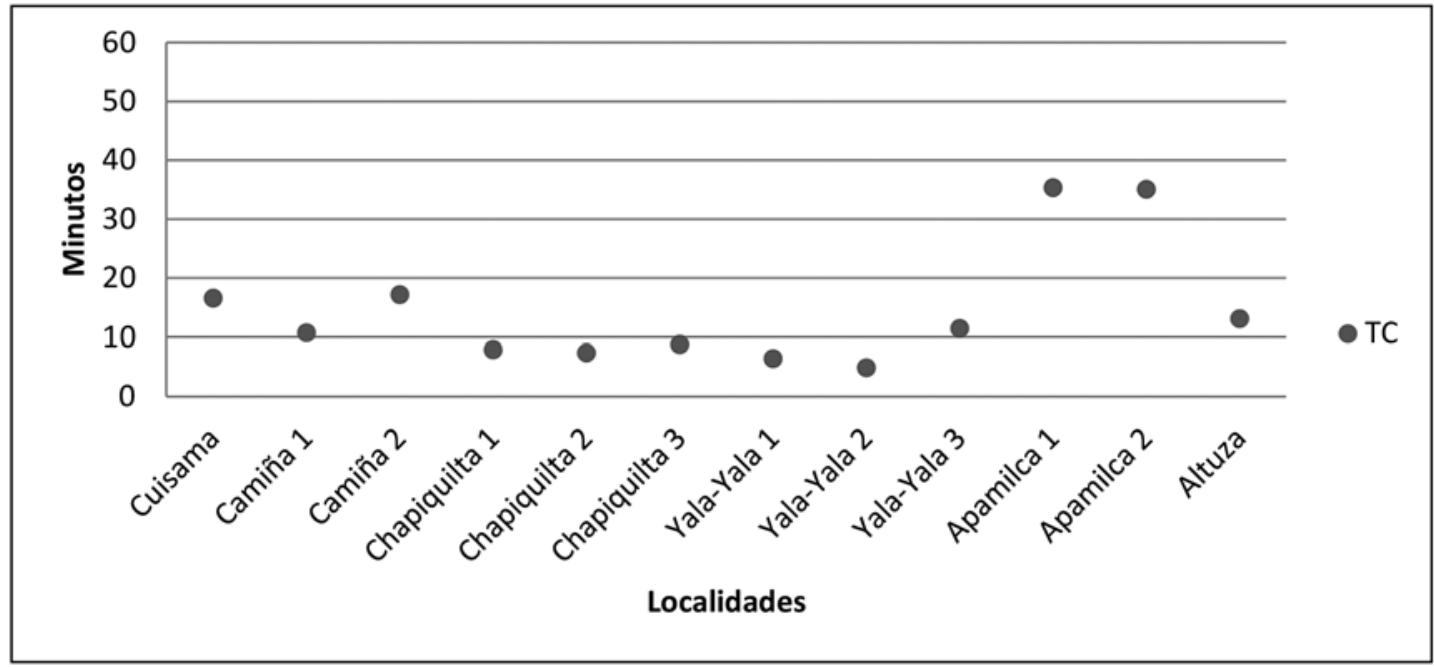

Figura 5. Tiempo de concentración $\left(\mathrm{T}_{\mathrm{c}}\right)$ microcuencas. Fuente: elaboración propia. 


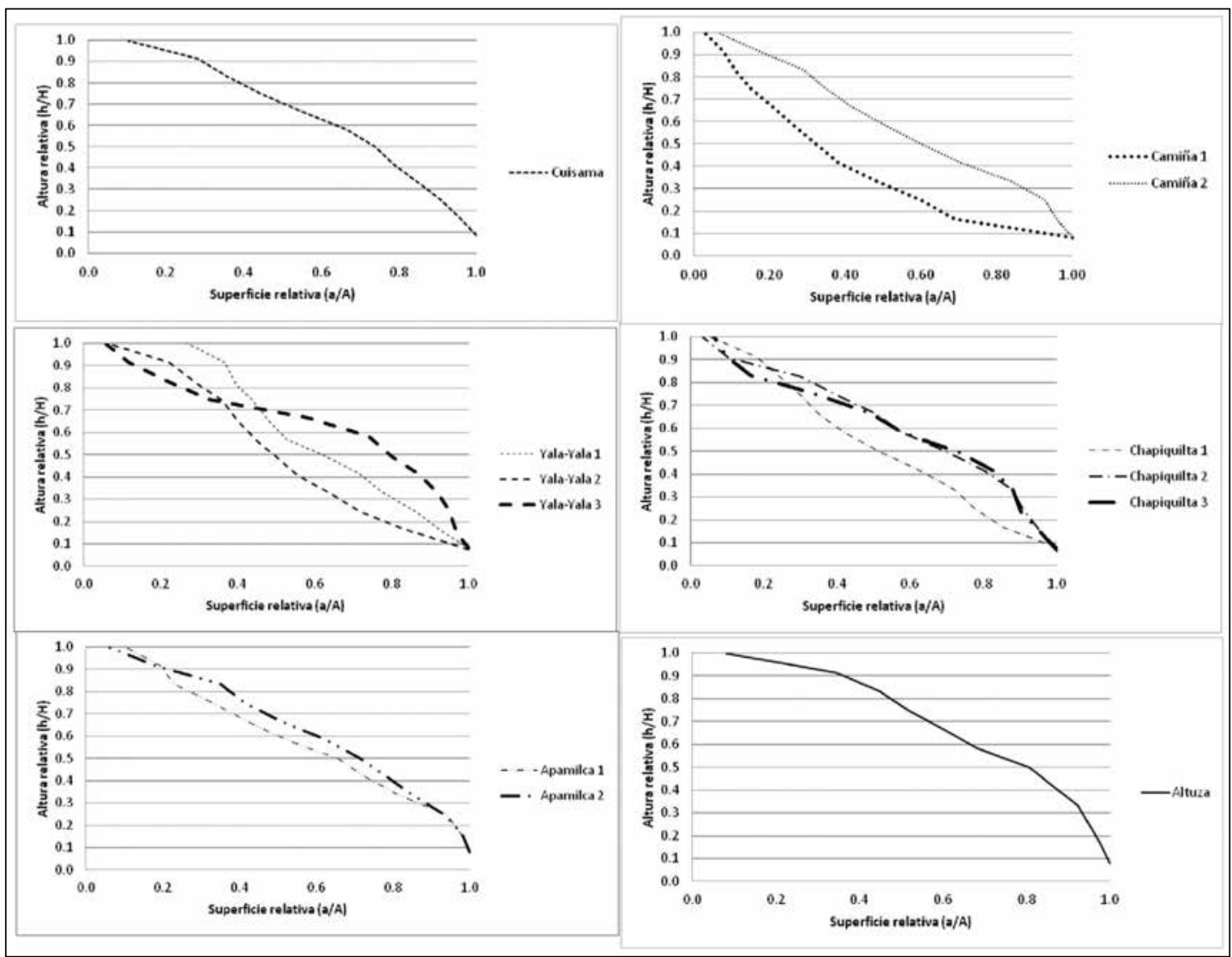

Figura 6. Curvas hipsométricas por microcuencas. Fuente: elaboración propia.

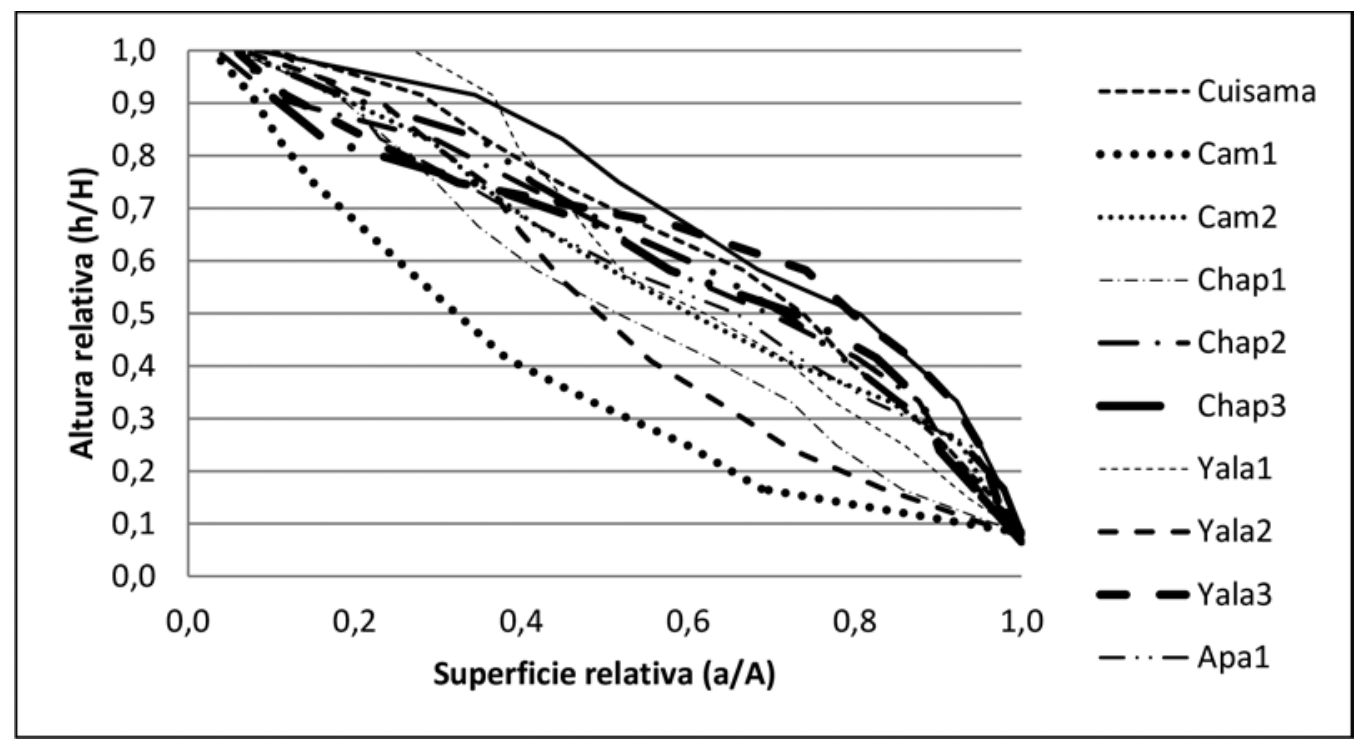

Figura 7. Curvas hipsométricas microcuencas. Fuente: Elaboración propia. 
geológicamente jóvenes y maduras, las primeras denominadas de meseta y las segundas de pie de montaña o piedemont, de acuerdo con lo establecido por Strahler (1952).

\section{Discusión y Conclusión}

Los resultados obtenidos del análisis morfométrico han permitido establecer las condiciones que poseían las microcuencas antes del evento natural ocurrido en marzo de 2012 en la quebrada de Camiña.

De acuerdo con estos resultados, las microcuencas poseían propiedades muy inestables, sobre todo las que demostraron altas pendientes promedio, importantes desniveles y superficies muy pequeñas. Resultan interesante las condiciones que poseían las cuencas de Camiña, Chapiquilta y Yala-Yala, pues las dos últimas fueron analizadas por medio de seis microcuencas, todas productoras de flujos de detritos en 2012, de las cuales solamente una sobrepasaba el $\mathrm{km}^{2}$. Los parámetros obtenidos demostraron valores de inestabilidad significativos como tamaños reducidos de superficie, índices de compacidad que no superan el valor 1,5 y densidades de drenaje muy altas, sumados los tiempos de concentración muy cortos que determinaron condiciones de erosión favorables. Casos menos extremos, pero que no dejan de ser significativos, lo constituyen las microcuencas de Apamilca que mostraron formas superficiales más alargadas que reducirían la erosión, algo que también se manifiesta en los tiempo de concentración; sin embargo, los altos desniveles y densidades de drenaje no posibilitan un estado de equilibrio erosivo para dichas microcuencas, más aún si consideramos que las curvas hipsométricas elaboradas demostraron una clara tendencia a ciclos erosivos en desequilibrio.

Es importante aclarar que los datos obtenidos para la microcuenca Camiña 1 no se centran en los fenómenos particulares ocurridos en las quebradas laterales del pueblo de Camiña, ya que estas quebradas tributarias al curso principal forman parte de un modelo de drenaje paralelo, implicando que los datos obtenidos no representen los flujos de detritos que se produjeron en dichas quebradas, porque el pueblo de Camiña se emplaza en la confluencia de los tres tributarios, perdiéndose por tanto información respecto de estos fenómenos particulares, y considerando además que los reportes de ONEMI señalan al pueblo de Camiña como el más afectado por los flujos de detritos. No obstante, los distintos parámetros morfométricos calculados, además del análisis hipsométrico, permiten determinar que aquella microcuenca se encontraba en un ciclo de erosión activo, que posibilitó el desarrollo de los flujos de detritos.

Las propiedades morfométricas de las microcuencas habrían favorecido la ocurrencia de flujos de detritos en marzo de 2012. De acuerdo con los resultados obtenidos, las microcuencas poseen propiedades que favorecen el libre escurrimiento de las corrientes superficiales efímeras y en tiempos muy reducidos, eventos que según la compacidad o formas de las cuencas se presentarían menos intensos en Apamilca. La menor probabilidad de pérdidas humanas, infraestructura u otras consecuencias negativas acaecidas en el pueblo de Apamilca responderían a su localización geográfica que no la expone a flujos que crucen el pueblo y también a los tiempos de concentración mayores, permitiendo contar con mayor tiempo para evacuar sectores bajo peligro.

Si bien todas las microcuencas presentaban condiciones propicias para la producción de movimientos en masa, destacan el caso de Camiña 1, Chapiquilta 1,2 y 3 , Yala-Yala 1 y 2 , debido a que presentan los tiempos de concentración más bajos, formas de cuenca compactas, superficies muy reducidas y muy altas densidades de drenaje. En el caso de Camiña 1 se suma un desnivel, que alcanza $648 \mathrm{~m}$. Esto convierte a los pueblos homónimos en unidades expuestas a importantes peligros de movimientos en masa

Los resultados obtenidos ratifican la información obtenida de los reportes de ONEMI en 2012, ya que las microcuencas más susceptibles a generar flujos violentos eran las de Camiña, Chapiquilta y Yala-Yala, lo que se apreció en la infraestructura devastada en estos pueblos, como también por el número de albergados en dichas localidades. Estos datos también coinciden con las zonas afectadas estudiadas por Méndez et al. (2013), referente a las localidades de Camiña y Chapiquilta; esta última devastada totalmente y con el mayor número de albergados (70 personas).

En síntesis, es posible señalar que las condiciones morfométricas de las microcuencas que componen la sección alta de la quebrada de Camiña son favorables para que el desencadenamiento de movimientos en masa en general. Lo anterior queda evidenciado con los desastres ocurridos el 13 de marzo del 2012. 
Los distintos parámetros analizados y el índice de compacidad permitieron verificar que los desastres ocurridos en las localidades de la parte alta de la quebrada fueron propiciados por las condiciones morfométricas de las microcuencas, que manifestaban condiciones propicias para el desarrollo de flujos de detritos.

Los valores obtenidos presentan una gran tendencia de las microcuencas a la acción erosiva en los cursos efímeros, lo que es ratificado por el análisis hipsométrico. En relación con esto último, cabe destacar que el total de las cuencas analizadas presentó una importante tendencia a ciclos erosivos en desequilibrio, traducido esto en una significativa acción erosiva capaz de transportar el material detrítico hacia las áreas de acumulación y terraza fluvial. En estas unidades se han establecido los asentamientos humanos y las actividades económicas de las comunidades que habitan el área de estudio, las que se ven notoriamente amenazadas cuando se presentan lluvias intensas y concentradas, tendencia que se ha acentuado en los últimos años.

La consideración de los índices aquí expuestos podrían contribuir a tomar decisiones en conjunto para mitigar el impacto de estos fenómenos, donde se debiesen integrar todos los actores (internos y externos), para conseguir una planificación acorde a las necesidades y objetivos de desarrollo endógeno de la quebrada, porque en escenarios de variabilidad climática se prevé una mayor ocurrencia de fenómenos de precipitación intensa y concentrada en cortos periodos.

\section{Referencias Citadas}

\section{Aceituno P}

1993 Aspectos generales del Altiplano sudamericano. Actas del II Simposio Internacional de Estudios Altiplánicos: 63-69.

Almorox, J., López, F., y Rafaelli, S.

2011 La degradación de los suelos por erosión hídrica. Editum. Ediciones de la Universidad de Murcia.

Campos D.

1992 Capítulo 2: Geomorfología de la cuenca. Procesos del Ciclo Hidrológico, editado por D. Campos, pp. 29. Editorial Universitaria Potosina, Potosí.

Gaillard J., Monteil C., Perrillat-Collomb A., Chaudhari S., Chaudhari M., Chaudhari O., Giazzi F. y Cadag J.

2013 Participatory 3-dimension mapping: A tool for encouraging multi-caste collaboration to climate change adaptation and disaster risk reduction. Applied Geography 45: 158-166.

Gaillard J. y Mercer J.

2013 From knowledge to action: Bridging gaps in disaster risk reduction. Progress in Human Geography 37 (1): 93-114.

Horton R.

1945 Erosional Development of Streams and their Drainage Basins; Hydrophysical Approach to Quantitative Morphology. Bulletin of the Geological Society of America 56: 275-370.

Ibáñez S., Moreno H. y Gisbert J.

Métodos para la determinación del tiempo de concentración (tc) de una cuenca hidrográfica. (30 noviembre). http:// riunet.upv.es/bitstream/handle/10251/10779/Tiempo\%20 de\%20concentraci\%C3\%B3n.pdf (4 de noviembre de 2013).

Instituto Nacional de Ecología

200 Análisis morfométrico de cuencas: caso de estudio del Parque Nacional Pico de Tancítaro. México. pp. 47.

Lacasse, S., y Nadim, F.

2008 Landslide Risk Assessment and Mitigation Strategy. En K. Sassa y P. Canuti (Eds.), Landslides-Disaster Risk Reduction (pp. 31-63). Berlín: Springer.
Li M., Chibber P.

2008 Overland Flow Time of Concentration on Very Flat Terrains. Transportation Research Record: Journal of the Transportation Research Board 2060: 133-140.

Llano, J.

1975 Mecánica de suelos. Barcelona: Editores Técnicos Asociados.

Méndez M., Romero H. y Sarricolea P.

2013 Vulnerabilidad social en el desierto de Atacama: aluviones del 13 de marzo de 2012. Quebrada de Camiña, Chile ( $19^{\circ}$ Lat S.). Presentada en el Encuentro de Geógrafos de América Latina, Perú.

Muñoz N. y Sepúlveda P.

1992 Estructuras compresivas con vergencia al oeste en el borde oriental de la Depresión Central, Norte de Chile (190 15'8). Andean Geology 19 (2): 241-247.

Oficina Nacional de Emergencias

2012a Reporte No 16-AZ: Informe de Incidente o Emergencia, Consolidado Informe Hidrometeorológico Alerta Roja, Provincia del Tamarugal, Región de Tarapacá, Curso de Acción. Oficina Nacional de Emergencia del Ministerio del Interior y Protección Civil. Manuscrito en posesión del autor.

Oficina Nacional de Emergencias

2012b Reporte N ${ }^{\circ}$ 16-A11: Informe de Incidente o Emergencia, Consolidado Informe Hidrometeorológico Alerta Roja, Provincia del Tamarugal, Región de Tarapacá, Curso de Acción. Oficina Nacional de Emergencia del Ministerio del Interior y Protección Civil. Manuscrito en posesión del autor.

Oficina Nacional de Emergencias

2012c Reporte No 16-A55: Informe de Incidente o Emergencia, Consolidado Informe Hidrometeorológico Alerta Roja, Provincia del Tamarugal, Región de Tarapacá, Curso de Acción. Oficina Nacional de Emergencia del Ministerio del Interior y Protección Civil. Manuscrito en posesión del autor. 
Pinto L., Hérail G. y Charrier R.

2004 Sedimentación sintectónica asociada a las estructuras neógenas en la precordillera de la zona de Moquella, Tarapacá (19¹5'S, Norte de Chile). Revista Geológica de Chile 31 (1): 19-44.

Pinto L., Hérail G., Sepúlveda S. y Krop P.

2008 A Neogene giant landslide in Tarapacá, northern Chile: A signal of instability of the westernmost Altiplano and palaeoseismicity effects. Geomorphology 102 (3-4): 532-531.

Quezada J., Cerda J. y Jensen A.

2010 Efectos de la tectónica y el clima en la configuración morfológica del relieve costero del Norte de Chile. Andean Geology 37 (1): 78-109.
Sharifi S. y Hosseini

2011 Methodology for Identifying the Best Equations for Estimating the Time of Concentration of Watersheds in a Particular Region. Journal of Irrigation and Drainage Engineering: 712-719.

SERNAGEOMIN

2003 Mapa Geológico de Chile. Versión digital. Servicio Nacional de Geología y Minería, Publicación Geológica Digital, 4 (CD-ROM, versión 1.0, 2003). Santiago.

Strahler A.

1952 Hypsometric (area-altitude) Analysis of Erosional Topography. Bulletin of the Geological Society of America 63: 1117-1142.

Walker, L. R., y Shiels, A. B.

2012 Landslide Ecology. New York Cambridge University Press. 